\title{
OPTIMAL PRODUCTION IN MONOPOLY PRICING: A STOCHASTIC AND DYNAMIC APPROACH
}

\author{
Francisco Venegas-Martínez* \\ Finance Department, Monterrey Institute of Technological and Higher Education, \\ Mexico City Campus
}

(Received 12 july 2001, accepted 21 january 2002)

\begin{abstract}
This paper is concerned with a special case of stochastic linear regulator that arises in optimal production planning in monopoly pricing. The state process consists of the inventory level and the demand rate (the latter taken as given). The inventory level involves both the production rate (control variable) and the demand rate. Several stochastic models for the demand are proposed and discussed. The state equation is a linear controlled stochastic equation driven by a Brownian motion. Costs are assumed to be quadratic and the LQG (Linear-QuadraticGaussian) approach is used to characterize optimal production-inventory policies. Finally, when possible, closed-form solutions are provided, otherwise approximate solutions are found by means of discrete-time linear systems.
\end{abstract}

\section{Resumen}

Este artículo se refiere a un caso especial de regulador lineal estocáśtico que surge en la planeación óptima de la producción en mercados monopólicos. El proceso de estado consiste en el nivel del inventario y la demanda (ésta última tomada como dada). A su vez, el nivel del inventario considera la producción (variable del control) y la demanda. Varios modelos estocásticos del comportamiento de la demanda se proponen y se discuten. La ecuación de estado es una ecuación lineal estocástica cóntrolada, la cual es conducida por un movimiento Browniano. Los costos se suponen cuadráticos y el modelo LQG (Linear-Quadratic-Gaussian) se utiliza para caracterizar las políticas óptimas de producción-inventario. Finalmente, se proporcionan soluciones analíticas cuando sea posible. En caso contrario, se proporcionan soluciones aproximadas a través del uso de sistemas lineales discretos.

Classification JEL: D42, D21, D24.

Keywords: Monopoly, Firm Behavior, Production Planning.

* Instituto 'Tecnológico y de Estudios Superiores de Monterrey, Campus Ciudad de México, Departamento de Finanzas, Calle del Puente 222, 14380 México, D. F., Telephone (52)54831861, E-mail: fvenegas@campus.ccm.itesm.mx

The author is very grateful to Roberto Ballinez for checking numerous mathematical details and two anonymous referees for their comments. 


\section{Introduction}

Determining production-inventory policies that provide minimum costs when a stochastic demand is taken as given, is of great importance in studying monopoly pricing. The literature regarding this field is scarce because of the presence of randomness, which introduces technical complexities. In this paper, the demand is assumed to be driven by a continuous Markovian processes with continuous paths (diffusion Markovian processes) ${ }^{1}$. Once a quadratic functional expressing production and inventory costs has been defined, the LQG approach is then used to characterize optimal production-inventory policies. Closed-form and approximate solutions, for several stochastic demand models taken as given, are found ${ }^{2}$.

This paper is organized as follows. In section 2, we propose several stochastic models for the demand. Under this framework, we study an oligopoly market with several plants operating jointly. In section 3 , we present a family of quadratic functionals expressing the inventory and production costs. It is here where the LQG approach is used to characterize optimal solutions. Through section 4, we provide closed-form and approximate solutions. Finally, in section 5 , we give a set of comments and remarks, as well as delimitations of the results obtained.

\section{Basic Demand Models}

The following models take into account the production of a single good and finite horizon. The multidimensional case for the production of several goods with independent demands will be treated later through subsections 2.3 and 2.4. The starting point of our analysis is the so-called balance equation:

$$
\sigma\left[I(t)-i_{0}\right]=\int_{0}^{t}[(\sigma U(s)+\mu)-X(s)] \mathrm{d} s,
$$

with the conditions $X(0)=x_{0}, I(0)=i_{0}, X(t) \geq \mu, 0 \leq t \leq T$. Here, $X(t)$ is the rate of demand at time $t ; \mu$ (the physical drift) is mean expected rate of demand; $\sigma$ is the constant diffusion coefficient or instantaneous volatility; $\sigma U(t)+\mu$ is the rate of production, scaled by $\sigma$, at time $t$, which is seen as a control function; and $\sigma I(t)$ is the level of inventory at time $t$, scaled by $\sigma$, too.

\subsection{First-Order Demand Models}

We first assume that the demand, $X(t)$, satisfies the first order stochastic differential equation of exponential growth (cf. Bensoussan et al. 1984).

$$
\mathrm{d}\left(\frac{X(t)-\mu}{\sigma}\right)=-a\left(\frac{X(t)-\mu}{\sigma}\right) \mathrm{d} t+\mathrm{d} Z(t), \quad 0 \leq t \leq T, \quad X(0)=x_{0},
$$

1 Problems in which the supply has random disturbances, for instance, the determination of optimal operation policies in dams, are studied in Hernández (1987), Pliska (1975), and Bather (1968).

2 As related references for the LQG approach we may mention, for instance: Arnold (1982), Elliott (1982), Bensoussan (1982), and Fleming and Rishel (1975). 
where $a$ is a constant different from zero and $Z(t) \sim \mathcal{N}(0, \mathrm{~d} t)$ is a standard Wiener process (standard Brownian motion, the continuous time version of a random walk). It is through the constant $a$ that it is possible to model a demand trend, increasing, for $-a>0$, or decreasing, for $-a<0$. The term $\mathrm{d} Z(t)$ appearing in (2.2) produces an effect of stochastic disturbances in the behavior of the demand. We may think of $X(t)-\mu$ in (2.2) as a process with average growth rate $-a$ and variance $\sigma^{2}$. By applying elementary stochastic calculus, it can be readily shown that the solution of $(2.2)$ is given by

$$
X(t)=\mu+e^{-a t}\left(x_{0}-\mu\right)+\int_{0}^{t} e^{-a(t-s)} \sigma \mathrm{d} Z(s) .
$$

Notice now that equations (2.1) and (2.2) can be written together in matrix form as

$$
\mathrm{d} Y(t)=[A Y(t)+B V(t)] \mathrm{d} t+C \mathrm{~d} Z(t), \quad Y(0)=y_{0},
$$

by setting

$$
\begin{gathered}
Y(t)=\left(\begin{array}{c}
X(t)-\mu \\
\sigma I(t)
\end{array}\right), \quad V(t)=\left(\begin{array}{c}
0 \\
U(t)
\end{array}\right), \quad A=\left(\begin{array}{cc}
-a & 0 \\
-1 & 0
\end{array}\right), \\
B=\left(\begin{array}{ll}
0 & 0 \\
0 & \sigma
\end{array}\right), \quad C=\left(\begin{array}{l}
\sigma \\
0
\end{array}\right), \quad y_{0}=\left(\begin{array}{c}
x_{0}-\mu \\
\sigma i_{0}
\end{array}\right) .
\end{gathered}
$$

Equation (2.3) is known as the equation of evolution of the linear regulator- (see, for instance, Fleming and Rishel, 1975). This equation plays an important role in many practical situations, mainly, in finance and economics.

\subsection{Second-Order Demand Models}

If we have an occurrence frequency between periods of high and low demand in $[0, T]$, then we may assume that the demand $X(t)$ satisfies the following secondorder stochastic differential equation (the stochastic harmonic oscillator):

$$
\frac{\mathrm{d}^{2}}{\mathrm{~d} t^{2}}\left(\frac{X(t)-\mu}{\sigma}\right)=-a\left(\frac{X(t)-\mu}{\sigma}\right)+\frac{\mathrm{d} Z(t)}{\mathrm{d} t}, \quad 0 \leq t \leq T,
$$

with initial conditions $X(0)=x_{0}$, and $\dot{X}(0)=\xi_{0}$. Here, the trend $a$ is a positive constant. The other constants $\mu$ and $\sigma$ are taken again as in equation(2.1). We warn the reader about the term $\mathrm{d} Z(t) / \mathrm{d} t$ in the above expression, this derivative does not exist and should be adequately interpreted as stochastic disturbances. By using elementary stochastic calculus, it can be shown that

$$
X(t)=\mu+\int_{0}^{t} e^{-a s}\left(x_{0}-\mu\right) \mathrm{d} s+\int_{0}^{t} \int_{0}^{u} e^{-a(u-s)} \sigma \mathrm{d} Z(s) \mathrm{d} u
$$

is solution of (2.4). Notice that, with a suitable change of variable, equation (2.4) can be writen as

$$
\left\{\begin{array}{l}
\mathrm{d}(X(t)-\mu)=\sigma L(t) \mathrm{d} t \\
\mathrm{~d} \sigma L(t)=-a(X(t)-\mu) \mathrm{d} t+\sigma \mathrm{d} Z(t), \\
X(0)=x_{0}, \quad L(0)=(1 / \sigma) \xi_{0}
\end{array}\right.
$$


In this case, equations (2.1) and (2.5) may be set in similar form to (2.3), by taking

$$
\begin{gathered}
Y(t)=\left(\begin{array}{c}
X(t)-\mu \\
\sigma I(t) \\
\sigma L(t)
\end{array}\right), \quad V(t)=\left(\begin{array}{c}
0 \\
U(t) \\
0
\end{array}\right), \quad A=\left(\begin{array}{ccc}
0 & 0 & 1 \\
-1 & 0 & 0 \\
-a & 0 & 0
\end{array}\right), \\
B=\left(\begin{array}{lll}
0 & 0 & 0 \\
0 & \sigma & 0 \\
0 & 0 & 0
\end{array}\right), \quad C=\left(\begin{array}{c}
0 \\
0 \\
\sigma
\end{array}\right), y_{0}=\left(\begin{array}{c}
x_{0}-\mu \\
\sigma i_{0} \\
\xi_{0}
\end{array}\right) .
\end{gathered}
$$

Suppose now that both frequency and demand decrease in time, then we may assume that the demand satisfies the equation for the stochastic damped oscillator:

$$
\frac{\mathrm{d}^{2}}{\mathrm{~d} t^{2}}\left(\frac{X(t)-\mu}{\sigma}\right)=-c \frac{\mathrm{d}}{\mathrm{d} t}\left(\frac{X(t)-\mu}{\sigma}\right)-a\left(\frac{X(t)-\mu}{\sigma}\right)+\frac{\mathrm{d} Z(t)}{\mathrm{d} t},
$$

with conditions $X(0)=x_{0}$ and $\dot{X}(0)=\xi_{0}$. Here, $c$ is a positive constant. In this case, taking the same change of variable as in (2.5), we get the following expression:

$$
\left\{\begin{array}{l}
\mathrm{d}(X(t)-\mu)=\sigma L(t) \mathrm{d} t \\
\mathrm{~d} \sigma L(t)=-c \sigma L(t) \mathrm{d} t-a(X(t)-\mu) \mathrm{d} t+\sigma \mathrm{d} Z(t), \\
X(0)=x_{0}, \quad L(0)=(1 / \sigma) \xi_{0} .
\end{array}\right.
$$

For this model, instead of having the matrix $A$, given before, we now have the matrix

$$
A_{0}=\left(\begin{array}{ccc}
0 & 0 & 1 \\
-1 & 0 & 0 \\
-a & 0 & -c
\end{array}\right) \text {. }
$$

We shall refer in what follows to (2.4) as the second-order model for $A$, and to (2.6) as the second-order model for $A_{0}$.

Finally, we present a model for coupled demands $X_{1}(t)$ and $X_{2}(t)$, both following second-order models, by assuming they satisfy the system:

$$
\begin{aligned}
\frac{\mathrm{d}^{2}}{\mathrm{~d} t^{2}}\left(\frac{X_{1}(t)-\mu_{1}}{\sigma_{1}}\right)= & -a_{1}\left(\frac{X_{1}(t)-\mu_{1}}{\sigma_{1}}\right)+a\left[\beta\left(\frac{X_{2}(t)-\mu_{2}}{\sigma_{2}}\right)\right. \\
& \left.-\left(\frac{X_{1}(t)-\mu_{1}}{\sigma_{1}}\right)\right]+\frac{\mathrm{d}}{\mathrm{d} t} Z_{1}(t), \\
\frac{\mathrm{d}^{2}}{d t^{2}}\left(\frac{X_{2}(t)-\mu_{2}}{\sigma_{2}}\right)= & -a_{2}\left(\frac{X_{2}(t)-\mu_{2}}{\sigma_{2}}\right)-a\left[\left(\frac{X_{2}(t)-\mu_{2}}{\sigma_{2}}\right)\right. \\
& \left.-\beta^{-1}\left(\frac{X_{1}(t)-\mu_{1}}{\sigma_{1}}\right)\right]+\frac{\mathrm{d}}{\mathrm{d} t} Z_{2}(t) .
\end{aligned}
$$


with initial conditions $X_{1}(0)=x_{1,0}, X_{2}(0)=x_{2,0}, \dot{X}_{1}(0)=\xi_{1,0}, \dot{X}_{2}(0)=\xi_{2,0}$. The constants $a, a_{1}, a_{2}$ and $\beta$ are all positive. Moreover, the constant $\beta$ is a technical coefficient that may be interpreted as the number of units of $X_{1}$ required to make a unit of $X_{2}$. The procesess $Z_{1}(t)$ and $Z_{2}(t)$ are assumed to be independent. We may obtain a similar equation to that of $(2.3)$ by setting

$$
\left\{\begin{aligned}
\mathrm{d} \sigma_{1} I_{1}(t)=\left[\sigma_{1} U_{1}(t)-\left(X_{1}(t)-\mu_{1}\right)\right] \mathrm{d} t, \quad X_{1}(t) \geq \mu_{1}, \\
\mathrm{~d} \sigma_{2} I_{2}(t)=\left[\sigma_{2} U_{2}(t)-\left(X_{2}(t)-\mu_{2}\right)\right] \mathrm{d} t, \quad X_{2}(t) \geq \mu_{2}, \\
\mathrm{~d}\left(X_{1}(t)-\mu_{1}\right)=\sigma_{1} L_{1}(t) \mathrm{d} t, \\
\mathrm{~d}\left(X_{2}(t)-\mu_{2}\right)=\sigma_{2} L_{2}(t) \mathrm{d} t, \\
\mathrm{~d} \sigma_{1} \sigma_{2} L_{1}(t)=-\left(a+a_{1}\right) \sigma_{2}\left(X_{1}(t)-\mu_{1}\right) \mathrm{d} t+a \sigma_{1} \beta\left(X_{2}(t)-\mu_{2}\right) \mathrm{d} t \\
\quad+\sigma_{1} \sigma_{2} \mathrm{~d} Z_{1}(t), \\
\mathrm{d} \sigma_{1} \sigma_{2} L_{2}(t)=-\left(a+a_{2}\right) \sigma_{1}\left(X_{2}(t)-\mu_{2}\right) \mathrm{d} t+a \sigma_{2} \beta^{-1}\left(X_{1}(t)-\mu_{1}\right) \mathrm{d} t \\
\quad+\sigma_{1} \sigma_{2} \mathrm{~d} Z_{2}(t), \\
\\
X_{k}(0)=x_{k, 0}, \quad I_{k}(0)=i_{k, 0}, \quad L_{k}(0)=\left(1 / \sigma_{k}\right) \xi_{k, 0}, \quad k=0,1,
\end{aligned}\right.
$$

and choosing

$$
\begin{aligned}
Y(t) & =\left(\begin{array}{c}
X_{1}(t)-\mu_{1} \\
X_{2}(t)-\mu_{2} \\
\sigma_{1} I_{1}(t) \\
\sigma_{2} I_{2}(t) \\
\sigma_{1} \sigma_{2} L_{1}(t) \\
\sigma_{1} \sigma_{2} L_{2}(t)
\end{array}\right), V(t)=\left(\begin{array}{c}
0 \\
0 \\
U_{1}(t) \\
U_{2}(t) \\
0 \\
0
\end{array}\right), y_{0}=\left(\begin{array}{c}
x_{1,0}-\mu_{1} \\
x_{2,0}-\mu_{2} \\
\sigma_{1} i_{1,0} \\
\sigma_{2} i_{2,0} \\
\sigma_{2} \xi_{1,0} \\
\sigma_{1} \xi_{2,0}
\end{array}\right), \\
A & =\left(\begin{array}{cccccc}
0 & 0 & 0 & 0 & \sigma_{2}^{-1} & 0 \\
0 & 0 & 0 & 0 & 0 & \sigma_{1}^{-1} \\
-1 & 0 & 0 & 0 & 0 & 0 \\
0 & -1 & 0 & 0 & 0 & 0 \\
-\left(a+a_{1}\right) \sigma_{2} & a \sigma_{1} \beta & 0 & 0 & 0 & 0 \\
a \sigma_{2} \beta^{-1} & -\left(a+a_{2}\right) \sigma_{1} & 0 & 0 & 0 & 0
\end{array}\right),
\end{aligned}
$$

$B$ a matrix with entries $B_{33}=\sigma_{1}, B_{44}=\sigma_{2}, B_{i j}=0$, otherwise, and $C$ a matrix with entries $C_{51}=\sigma_{1} \sigma_{2}, C_{62}=\sigma_{1} \sigma_{2}, C_{i j}=0$, otherwise. In this case, $Z(t)$ is a vector of independent Wiener processes, containing as the first two components $Z_{1}(t)$ and $Z_{2}(t)$.

\subsection{A Monopoly Model with Production Plants Operating Jointly}

For the sake of simplicity, we first consider only two plants operating jointly; the ideas involved in the proposed model can be easily extended to more plants. Assume that plants $P_{1}$ and $P_{2}$ produce the same generic good, and that $P_{1}$ has greater capability of production than $P_{2}$. Suppose also that from the total production of $P_{1}, U_{1}(t)$, a constant proportion, say $(1-\alpha) U_{1}(t)$, for a fixed $\alpha \in(0,1)$, is transfered to plant $P_{2}$, so that the latter is able to satisfy its 
demand. If $X_{1}(t)$ and $X_{2}(t)$ stand for the demands at each plant. Then the balance equations turn out to be:

$$
\left\{\begin{array}{l}
\mathrm{d} \sigma_{1} I_{1}(t)=\left[\sigma_{1} \alpha U_{1}(t)-\left(X_{1}(t)-\mu_{1}\right)\right] \mathrm{d} t, \quad X_{1}(t) \geq \mu_{1}, \\
\mathrm{~d} \sigma_{2} I_{2}(t)=\left\{\sigma_{2}\left[(1-\alpha) U_{1}(t)+U_{2}(t)\right]-\left(X_{2}(t)-\mu_{2}\right)\right\} \mathrm{d} t, \quad X_{2}(t) \geq \mu_{2}, \\
X_{1}(0)=x_{1,0}, \quad X_{2}(0)=x_{2,0}, \quad I_{1}(0)=i_{1,0}, \quad I_{2}(0)=i_{2,0} .
\end{array}\right.
$$

Assuming now that $X_{1}(t)$ and $X_{2}(t)$ are independent processes and that, for instance, they both follow a first-order model with coefficients $a$ and $b$, respectively, then taking

$$
\begin{gathered}
Y(t)=\left(\begin{array}{c}
X_{1}(t)-\mu_{1} \\
X_{2}(t)-\mu_{2} \\
\sigma_{1} I_{1}(t), \\
\sigma_{2} I_{2}(t)
\end{array}\right), V(t)=\left(\begin{array}{c}
0 \\
0 \\
U_{1}(t) \\
U_{2}(t)
\end{array}\right), A=\left(\begin{array}{cccc}
-a & 0 & 0 & 0 \\
0 & -b & 0 & 0 \\
-1 & 0 & 0 & 0 \\
0 & -1 & 0 & 0
\end{array}\right), \\
B=\left(\begin{array}{cccc}
0 & 0 & 0 & 0 \\
0 & 0 & 0 & 0 \\
0 & 0 & \alpha \sigma_{1} & 0 \\
0 & 0 & (1-\alpha) \sigma_{2} & \sigma_{2}
\end{array}\right), C=\left(\begin{array}{cccc}
\sigma_{1} & 0 & 0 & 0 \\
0 & \sigma_{2} & 0 & 0 \\
0 & 0 & 0 & 0 \\
0 & 0 & 0 & 0
\end{array}\right), \\
y_{0}=\left(\begin{array}{l}
x_{1,0}-\mu_{1} \\
x_{2,0}-\mu_{2} \\
\sigma_{1} i_{1,0} \\
\sigma_{2} i_{2,0}
\end{array}\right),
\end{gathered}
$$

we get a similar expression to $(2.3) \cdot$ with $Z(t)=\left[Z_{1}(t), Z_{2}(t), Z_{3}(t), Z_{4}(t)\right]^{\prime}$, (an apostrophe will denote the transpose operation), is a vector of independent Wiener processes. The processes $Z_{3}(t)$ and $Z_{4}(t)$ have been merely introduced for technical convenience.

The previous arguments have been applied when both plants produce the same good. However, when the plants produce different goods, say, $g_{1}$ and $g_{2}$, so that $g_{1}$ is required to produce $g_{2}$, then we just need to multiply the transfered quantity by a technical coefficient $\beta$ to transform units of $g_{1}$ into units of $g_{2}$. Thus, either assuming that the transformed production, $\beta(1-\alpha) U_{1}(t)$, is additional to some production $U_{2}(t)$, which is obtained by $P_{2}$ without using resources from $P_{1}$, we have that the balance equation for $P_{2}$ is

$$
\mathrm{d} \sigma_{2} I_{2}(t)=\left\{\sigma_{2}\left[\beta(1-\alpha) U_{1}(t)+U_{2}(t)\right]-\left(X_{2}(t)-\mu_{2}\right)\right\} \mathrm{d} t,
$$

or assuming that all the production of $P_{2}$ is the transformed production, then the balance equation for $P_{2}$ simple becomes

$$
\mathrm{d} \sigma_{2} I_{2}(t)=\left\{\sigma_{2} \beta(1-\alpha) U_{1}(t)-\left(X_{2}(t)-\mu_{2}\right)\right\} \mathrm{d} t .
$$




\subsection{Multi-dimensional Demand Models}

Consider a demand vector for $n$ goods, say, $X(t)=\left[X_{1}(t), X_{2}(t), \ldots, X_{n}(t)\right]^{\prime}$, which consists of independent components. In this case, the system of balance equations and the multivariate first-order model have the following extensions:

$$
\left\{\begin{array}{l}
\mathrm{d} \Sigma I(t)=[\Sigma U(t)-(X(t)-\mu)] \mathrm{d} t \\
\mathrm{~d}(X(t)-\mu)=-\Lambda(X(t)-\mu)+\Sigma \mathrm{d} Z(t), \\
X(0)=x_{0}, \quad I(0)=i_{0}
\end{array}\right.
$$

where $\Sigma=\left[\sigma_{i}\right]_{i=1}^{n}, \sigma_{i}>0, i=1,2, \ldots, n$, and $\Lambda=\left[\lambda_{i}\right]_{i=1}^{n}, \lambda_{i} \neq 0, i=1,2, \ldots, n$, are diagonal matrices; $Z(t)$ is a random vector containing independent Wiener processes; $\Sigma U(t)+\mu$ is the vector of scaled productions; and $\Sigma I(t)$ is the vector of scaled inventories. The (unique) solution, in vector form, of the stochastic differential equation in (2.9) is given by

$$
X(t)=\mu+e^{-\Lambda t}\left(x_{0}-\mu\right)+\int_{0}^{t} e^{-\Lambda(t-s)} \Sigma \mathrm{d} Z(s) .
$$

According to the extension of (2.3) to the multidimensional case, we have that the dimension of the corresponding vector $Y(t)=[X(t)-\mu, \Sigma I(t)]^{\prime}$ is $2 n$. The corresponding matrices $A, B$, and $C$ follow a similar pattern to that of the matrices from subsection 2.3 .

The analyses for the case in which the demand model is of second order, and even the mixed case (first and second-order models interacting) have no essential differences from the analysis done before, since components are individually treated.

\section{Quadratic Cost Functionals: LQG Theory}

Next, we introduce a family of quadratic functionals to evaluate the total cost of production and inventory, when an initial condition $Y(t)=y$ is given and a control (production) function $V(y, t)$ is used. The family of functionals with which we shall be concerned, $\left\{J_{P, Q, M}^{V}\right\}$, is a function of matrices $P, Q$ and $M$, where $P$ and $M$ are both symmetric positive semidefinite, and $Q$ is symmetric positive definite. These matrices keep technical coefficients for costs. In practice, very often, these costs have a quadratic behavior, (e.g. costs of generation of electric energy), being proportional to the functional

$$
\begin{aligned}
J_{P, Q, M}^{V}(y, T-t)= & \mathrm{E}\left\{\int_{t}^{T}[\langle Y(s), P Y(s)\rangle+\langle V(s), Q V(s)\rangle] \mathrm{d} s \mid \Im_{y, V}\right\} \\
& +\langle Y(T), M Y(T)\rangle,
\end{aligned}
$$

where

$$
Y(s)=y_{0}+\int_{0}^{s}[A Y(u)+B V(u)] \mathrm{d} u+\int_{0}^{s} C \mathrm{~d} Z(u),
$$


here, $\langle\cdot, \cdot\rangle$ is the usual inner product in the Euclidean space $\mathbb{R}^{n}, \Im_{y, V}$ denotes the sets $\{Y(t)=y$ is given, and the control $V(y, t)$ is used $\}$, and $\langle Y(T), M Y(T)\rangle$ is a terminal cost.

We notice that the integral in (3.1) takes a stochastic behavior because of its relation with the linear regulator. From now on, we shall leave out the subindexes $P, Q$ and $M$, and denote $\tau=T-t=$ "time to go", $0 \leq \tau \leq T$ (so $t=$ "absolute time"). The LQG approach characterizes $V^{*}(y, t)$, so that

$$
J(y, \tau)=J^{V^{*}}(y, \tau)=\min _{\substack{V(y, s) \\ t \leq s \leq T}} J^{V}(y, \tau)
$$

where the minimum is taken over all admissible controls $V(y, s)$ in $[t, T]$ (v.g. controls satisfying Lipschitz conditions uniformly in the variable $y$ ). Therefore, the LQG approach leads us to

$$
V^{*}(y, t)=-Q^{-1} B^{\prime} S^{*}(\tau) y
$$

where $S^{*}(\tau)$ is a symmetric positive definite matrix which solves

$$
-\frac{\mathrm{d}}{\mathrm{d} \tau} S(\tau)+A^{\prime} S(\tau)+S(\tau) A+P-S(\tau) B Q^{-1} B^{\prime} S(\tau)=0,
$$

with the condition $S(0)=M$. Equation (3.4) is known as the Ricatti differential equation, which may be seen as the adjoin equation of the optimal trajectory $S(\tau)$. A meaningful fact about this equation is that, under very general conditions, is that it has a bounded solution on any finite interval (see, for instance, Fleming and Rishel, 1975).

\section{Optimal Characterization of Production-Inventory Policies}

In this section, as immediate consequence of (3.3), we shall characterize optimal production-inventory policies for the discussed models, when the cost technical coefficients for $P, Q$, and $M$ do not include crossed terms, that is, when $Q=$ $M=I$ and $P$ is a diagonal matrix with non-negative terms. When $P, Q$ and $M$ are arbitrary diagonal matrices, the analysis will be basically the same. The general case, when $P, Q$ and $M$ are taken as in (3.1), will just take a little more of effort, making the notation cumbersome.

\subsection{Optimal Production-Inventory Policies for the First-Order De- mand Model}

For the first-order model introduced in subsection 2.1, if we take $Q=M=I$ and $P_{22}=1, P_{i j}=0$, otherwise, it follows that the optimal control satisfies

$$
U^{*}(y, t)=-\sigma\left[S_{21}^{*}(\tau) y_{1}+S_{22}^{*}(\tau) y_{2}\right], Y(t)=y, \tau=T-t,
$$


where $S_{21}^{*}(\tau)$ and $S_{22}^{*}(\tau)$ are solutions of the system of differential equations

$$
\left\{\begin{array}{l}
\frac{\mathrm{d}}{\mathrm{d} \tau} S_{21}(\tau)=-\left[a+\sigma^{2} S_{22}(\tau)\right] S_{21}(\tau)-S_{22}(\tau) \\
\frac{\mathrm{d}}{\mathrm{d} \tau} S_{22}(\tau)=1-\sigma^{2} S_{22}^{2}(\tau), \quad S_{22}(0)=1, \quad S_{21}(0)=0 .
\end{array}\right.
$$

The solution of system (4.1), provided $\sigma \neq 1$, is given by

$$
\left\{\begin{array}{l}
S_{22}^{*}(\tau)=\frac{1}{\sigma}\left[\frac{(1+\sigma)(1-\sigma)^{-1} e^{2 \sigma \tau}-1}{(1+\sigma)(1-\sigma)^{-1} e^{2 \sigma \tau}+1}\right] \\
S_{21}^{*}(\tau)=-\int_{0}^{\tau} S_{22}^{*}(s) \exp \{-a(\tau-s)-[Q(\tau)-Q(s)]\} \mathrm{d} s
\end{array}\right.
$$

where

$$
Q(\tau)=\sigma^{2} \int_{0}^{\tau} S_{22}^{*}(u) \mathrm{d} u
$$

When $\sigma=-1$, we get $S_{22}^{*}(\tau) \equiv-1$. In this case, $S_{21}^{*}(\tau)=(1-a)^{-1}(1-$ $\left.e^{-\tau(1-a)}\right)$ for $a \neq 1$, and $S_{21}^{*}(\tau)=-\tau$ for $a=1$.

We may provide an approximate solution for $S_{21}(\tau)$, when $\tau$ is small, as follows: observe that, if $\tau$ is small, so is $\tau-s$, since $0 \leq s \leq \tau$. Therefore, $Q(\tau)-Q(s) \approx Q^{\prime}(\tau)(\tau-s)$, then, for small $\tau$, we may well use the approximation

$$
S_{21}^{*}(\tau) \approx-\int_{0}^{\tau} S_{22}^{*}(s) e^{-(\tau-s)\left[a+\sigma^{2} S_{22}^{*}(\tau)\right]} \mathrm{d} s
$$

\subsection{Optimal Production-Inventory Policies for the Second-Order De- mand Models}

When the demand follows the second-order model for $A, Q=M=I$ and $P_{22}=1, P_{i j}=0$, otherwise, the optimal control has the form

$$
U^{*}(y, t)=-\sigma\left[S_{21}^{*}(\tau) y_{1}+S_{22}^{*}(\tau) y_{2}+S_{23}^{*}(\tau) y_{3}\right], Y(t)=y, \tau=T-t,
$$

where $S_{22}^{*}(\tau)$ is taken again as in $(4.2)$, and $\left(S_{21}^{*}(\tau), S_{23}^{*}(\tau)\right)$ is solution of the system

$$
\left\{\begin{array}{l}
\frac{\mathrm{d}}{\mathrm{d} t} S_{21}(\tau)=-\left[1+\sigma^{2} S_{21}(\tau)\right] S_{22}^{*}(\tau)-a S_{23}(\tau), \\
\frac{\mathrm{d}}{\mathrm{d} t} S_{23}(\tau)=S_{21}(\tau)-\sigma^{2} S_{22}^{*}(\tau) S_{23}(\tau) \\
S_{21}(0)=S_{23}(0)=0
\end{array}\right.
$$

If we suppose a parameter value $a=1$, the joint solution of (4.5) turns out to be (see, for instance, Hirsh 1974, ch. 5):

$$
\left\{\begin{array}{l}
S_{21}^{*}(\tau)=-\int_{0}^{\tau} S_{22}^{*}(s) e^{-[Q(\tau)-Q(s)]} \cos (\tau-s) \mathrm{d} s \\
S_{23}^{*}(\tau)=-\int_{0}^{\tau} S_{22}^{*}(s) e^{-[Q(\tau)-Q(s)]} \sin (\tau-s) \mathrm{d} s
\end{array}\right.
$$


where we have taken $Q(\tau)$ as in (4.3). Approximate solutions for $S_{21}(\tau)$ and $S_{23}(\tau)$, when $\tau$ is small, can be found by using $\sin (\tau-s) \approx \tau-s$ and $\cos (\tau-s) \approx$ $1-(1 / 2)(\tau-s)^{2}$, so

$$
\left\{\begin{array}{l}
S_{21}^{*}(\tau) \approx-\int_{0}^{\tau} S_{22}^{*}(s) e^{-(\tau-s) \sigma^{2} S_{22}^{*}(\tau)}\left[1-\frac{1}{2}(\tau-s)^{2}\right] \mathrm{d} s \\
S_{23}^{*}(\tau) \approx-\int_{0}^{\tau} S_{22}^{*}(s) e^{-(\tau-s) \sigma^{2} S_{22}^{*}(\tau)}(\tau-s) \mathrm{d} s .
\end{array}\right.
$$

Next, we shall provide approximate solutions for the second-order model for $A_{0}$, the optimal control also satisfies $(4.4)$, but $\left[S_{21}^{*}(\tau), S_{22}^{*}(\tau), S_{23}^{*}(\tau)\right]$ are now solutions of the system

$$
\left(\begin{array}{l}
S_{21}(\tau) \\
S_{23}(\tau)
\end{array}\right)=H^{*}(\tau)\left(\begin{array}{c}
S_{21}(\tau) \\
S_{23}(\tau)
\end{array}\right)-\left(\begin{array}{c}
S_{22}^{*}(\tau) \\
0
\end{array}\right)
$$

where

$$
H^{*}(\tau)=\left(\begin{array}{cc}
-\sigma^{2} S_{22}^{*}(\tau) & -a \\
1 & -c-\sigma_{2} S_{22}^{*}(\tau)
\end{array}\right)
$$

and $S_{22}^{*}(\tau)$ is taken as in (4.2).

In order to obtain approximate solutions of (4.6), we proceed to apply Euler's method, getting the following discrete-time linear system:

$$
\left(\begin{array}{c}
S_{21}\left(\tau_{k+1}\right) \\
S_{23}\left(\tau_{k+1}\right)
\end{array}\right)=\left[\frac{T}{N} H^{*}\left(\tau_{k}\right)+I\right]\left(\begin{array}{c}
S_{21}\left(\tau_{k}\right) \\
S_{23}\left(\tau_{k}\right)
\end{array}\right)-\frac{T}{N}\left(\begin{array}{c}
S_{22}^{*}\left(\tau_{k}\right) \\
0
\end{array}\right),
$$

where $\tau_{k}=k \frac{T}{N}, k=0,1,2, \ldots, N$, and the matrix $H^{*}\left(\tau_{k}\right)$ is given by

$$
H^{*}\left(\tau_{k}\right)=\left(\begin{array}{cc}
-\sigma^{2} S_{22}^{*}\left(\tau_{k}\right) & -a \\
1 & -c-\sigma_{2} S_{22}^{*}\left(\tau_{k}\right)
\end{array}\right)
$$

The solution of (4.7) is found as

$$
\begin{aligned}
\left(\begin{array}{l}
S_{21}^{*}\left(\tau_{k+1}\right) \\
S_{23}^{*}\left(\tau_{k+1}\right)
\end{array}\right) & =-\frac{T}{N}\left\{\prod_{i=1}^{k}\left(\frac{T}{N} H^{*}\left(\tau_{i}\right)+I\right)\right\}\left(\begin{array}{l}
1 \\
0
\end{array}\right)-\frac{T}{N}\left(\begin{array}{c}
S_{22}^{*}\left(\tau_{k}\right) \\
0
\end{array}\right) \\
& -\frac{T}{N} \sum_{i=1}^{k}\left[\left(\frac{T}{N} H^{*}\left(\tau_{k}\right)+I\right) \cdots\left(\frac{T}{N} H^{*}\left(\tau_{i+1}\right)+I\right)\right]\left(\begin{array}{c}
S_{22}^{*}\left(\tau_{i}\right) \\
0
\end{array}\right) .
\end{aligned}
$$

Finally, we consider the model for coupled demands when $Q=M=I$ and $P_{33}=P_{44}=1, P_{i j}=0$, otherwise. In this case, the optimal controls are given by

$$
\left\{\begin{array}{l}
U_{1}^{*}(y, t)=-\sigma_{1} \sum_{j=1}^{4} S_{3 j}^{*}(\tau) y_{j}, \\
U_{2}^{*}(y, t)=-\sigma_{2} \sum_{j=1}^{4} S_{4 j}^{*}(\tau) y_{j},
\end{array}\right.
$$


where $Y(t)=y$ and $\tau=T-t$. Here, $\left[S_{3 j}^{*}\right]_{j=1}^{4}$ and $\left[S_{4 j}^{*}\right]_{j=1}^{4}$ are the joint solution of the system of differential equations, which, for convenience, has been separated into three groups of equations, as follows:

$$
\begin{aligned}
& \left\{\begin{array}{l}
\frac{\mathrm{d}}{\mathrm{d} \tau} S_{33}(\tau)=1-\sigma_{1}^{2} S_{33}^{2}(\tau)-\sigma_{2}^{2} S_{43}^{2}(\tau), \\
\frac{\mathrm{d}}{\mathrm{d} \tau} S_{44}(\tau)=1-\sigma_{1}^{2} S_{43}^{2}(\tau)-\sigma_{2}^{2} S_{44}^{2}(\tau), \\
\frac{\mathrm{d}}{\mathrm{d} \tau} S_{43}(\tau)=-\sigma_{1}^{2} S_{43}(\tau) S_{33}(\tau)-\sigma_{2}^{2} S_{44}(\tau) S_{43}(\tau),
\end{array}\right. \\
& \int \frac{\mathrm{d}}{\mathrm{d} \tau} S_{31}(\tau)=-S_{33}(\tau)-\left(a+a_{1}\right) \sigma_{2} S_{35}(\tau)+a \sigma_{2} \beta^{-1} S_{36}(\tau)-\sigma_{1}^{2} S_{31}(\tau) S_{33}(\tau) \\
& -\sigma_{2}^{2} S_{41}(\tau) S_{43}(\tau) \\
& \frac{\mathrm{d}}{\mathrm{d} \tau} S_{32}(\tau)=-S_{34}(\tau)+a \sigma_{1} \beta S_{35}(\tau)-\left(a+a_{2}\right) \sigma_{1} S_{36}(\tau)-\sigma_{1}^{2} S_{32}(\tau) S_{33}(\tau) \\
& -\sigma_{2}^{2} S_{42}(\tau) S_{43}(\tau) \\
& \frac{\mathrm{d}}{\mathrm{d} \tau} S_{35}(\tau)=\sigma_{2}^{-1} S_{31}(\tau)-\sigma_{1}^{2} S_{33}(\tau) S_{35}(\tau)-\sigma_{2}^{2} S_{45}(\tau) S_{43}(\tau), \\
& \frac{\mathrm{d}}{\mathrm{d} \tau} S_{36}(\tau)=\sigma_{1}^{-1} S_{32}(\tau)-\sigma_{1}^{2} S_{36}(\tau) S_{33}(\tau)-\sigma_{2}^{2} S_{46}(\tau) S_{43}(\tau), \\
& \begin{aligned}
\frac{\mathrm{d}}{\mathrm{d} \tau} S_{41}(\tau)= & -S_{34}(\tau)-\left(a+a_{1}\right) \sigma_{2} S_{45}(\tau)+a \sigma_{2} \beta^{-1} S_{46}(\tau)-\sigma_{1}^{2} S_{31}(\tau) S_{34}(\tau) \\
& -\sigma_{2}^{2} S_{41}(\tau) S_{44}(\tau),
\end{aligned} \\
& \left\{\begin{aligned}
\frac{\mathrm{d}}{\mathrm{d} \tau} S_{42}(\tau)= & -S_{44}(\tau)-a \sigma_{2} \beta S_{45}(\tau)-\left(a+a_{2}\right) \sigma_{1} S_{46}(\tau)-\sigma_{1}^{2} S_{32}(\tau) S_{34}(\tau) \\
& -\sigma_{2}^{2} S_{42}(\tau) S_{44}(\tau),
\end{aligned}\right. \\
& \frac{\mathrm{d}}{\mathrm{d} \tau} S_{45}(\tau)=\sigma_{2}^{-1} S_{41}(\tau)-\sigma_{1}^{2} S_{35}(\tau) S_{34}(\tau)-\sigma_{2}^{2} S_{45}(\tau) S_{44}(\tau), \\
& \frac{\mathrm{d}}{\mathrm{d} \tau} S_{46}(\tau)=\sigma_{1}^{-1} S_{42}(\tau)-\sigma_{1}^{2} S_{36}(\tau) S_{34}(\tau)-\sigma_{2}^{2} S_{46}(\tau) S_{44}(\tau),
\end{aligned}
$$

with the conditions $S_{33}(0)=S_{44}(0)=1$, and $S_{31}(0)=S_{32}(0)=S_{41}(0)=$ $S_{42}(0)=S_{43}(0)=0$. We shall provide solutions when $\sigma_{1}=\sigma_{2}=\sigma$. In this case, $S_{33} \equiv S_{44}$, and the first three-equation group becomes

$$
\left\{\begin{array}{l}
\frac{\mathrm{d}}{\mathrm{d} \tau} S_{33}(\tau)=1-\sigma^{2}\left[S_{33}^{2}(\tau)+S_{43}^{2}(\tau)\right] \\
\frac{\mathrm{d}}{\mathrm{d} \tau} S_{43}(\tau)=-2 \sigma^{2} S_{33}(\tau) S_{43}(\tau)
\end{array}\right.
$$

from where

$$
\left\{\begin{array}{l}
\frac{\mathrm{d}}{\mathrm{d} \tau}\left[S_{33}(\tau)+S_{43}(\tau)\right]=1-\sigma^{2}\left[S_{33}(\tau)+S_{43}(\tau)\right]^{2}, \\
\frac{\mathrm{d}}{\mathrm{d} \tau}\left[S_{33}(\tau)-S_{43}(\tau)\right]=1-\sigma^{2}\left[S_{33}(\tau)-S_{43}(\tau)\right]^{2},
\end{array}\right.
$$


so that, $S_{33}+S_{34} \equiv S_{33}-S_{34}$, therefore $S_{34}^{*} \equiv 0$ and so $S_{33}^{*}$ and $S_{44}^{*}$ both coincide with $S_{22}^{*}$ in (4.2). The second four-equation group may be writen now as

$$
\frac{\mathrm{d}}{\mathrm{d} t}\left(\begin{array}{c}
S_{31}(\tau) \\
S_{32}(\tau) \\
S_{35}(\tau) \\
S_{36}(\tau)
\end{array}\right)=\left[-\sigma^{2} S_{33}^{*}(\tau) I+K\right]\left(\begin{array}{c}
S_{31}(\tau) \\
S_{32}(\tau) \\
S_{35}(\tau) \\
S_{36}(\tau)
\end{array}\right)+\left(\begin{array}{c}
-S_{33}^{*}(\tau) \\
0 \\
0 \\
0
\end{array}\right)
$$

where

$$
K=\left(\begin{array}{cccc}
0 & 0 & -\left(a+a_{1}\right) \sigma & a \sigma \beta^{-1} \\
0 & 0 & a \sigma \beta & -\left(a+a_{2}\right) \sigma \\
\sigma^{-1} & 0 & 0 & 0 \\
0 & \sigma^{-1} & 0 & 0
\end{array}\right)
$$

By applying Euler's method to (4.8), we obtain the following discrete-time linear system, with $\tau_{k}=k(T / N), k=0,1,2, \ldots, N$ :

$$
\left(\begin{array}{l}
S_{31}\left(\tau_{k+1}\right) \\
S_{32}\left(\tau_{k+1}\right) \\
S_{35}\left(\tau_{k+1}\right) \\
S_{36}\left(\tau_{k+1}\right)
\end{array}\right)=\left[\frac{T}{N}\left(K-\sigma^{2} S_{33}^{*}\left(\tau_{k}\right)\right)+I\right]\left(\begin{array}{c}
S_{31}\left(\tau_{k}\right) \\
S_{32}\left(\tau_{k}\right) \\
S_{35}\left(\tau_{k}\right) \\
S_{36}\left(\tau_{k}\right)
\end{array}\right)-\frac{T}{N} S_{33}^{*}\left(\tau_{k}\right) \mathbf{e}_{\mathbf{1}}
$$

The solutions of (4.9) is

$$
\begin{gathered}
\left(\begin{array}{c}
S_{31}^{*}\left(\tau_{k+1}\right) \\
S_{32}^{*}\left(\tau_{k+1}\right) \\
S_{35}^{*}\left(\tau_{k+1}\right) \\
S_{36}^{*}\left(\tau_{k+1}\right)
\end{array}\right)=-\frac{T}{N}\left\{\prod_{i=1}^{k}\left[\frac{T}{N}\left(K-\sigma^{2} S_{33}^{*}\left(\tau_{i}\right) I\right)+I\right]\right\} \mathbf{e}_{\mathbf{1}}-\frac{T}{N} S_{33}^{*}\left(\tau_{k}\right) \mathbf{e}_{\mathbf{1}} \\
-\frac{T}{N} \sum_{i=1}^{k}\left\{\left[\frac{T}{N}\left(K-\sigma^{2} S_{33}^{*}\left(\tau_{k}\right) I\right)+I\right] \cdots\left[\frac{T}{N}\left(K-\sigma^{2} S_{33}^{*}\left(\tau_{i+1}\right) I\right)+I\right]\right\} S_{33}^{*}\left(\tau_{i}\right) \mathbf{e}_{1}
\end{gathered}
$$

where $\mathbf{e}_{\mathbf{1}}=[1,0,0,0]^{\prime}$. The third four-equation group can be similarly treated.

\subsection{Optimal Production-Inventory Policies for the Model of Plants Operating Jointly}

For the monopoly model with plants operating jointly, we have that the optimal controls, provided $Q=M=I$ and $P_{33}=P_{44}=1, P_{i j}=0$, otherwise, satisfy

$$
\left\{\begin{array}{l}
U_{1}^{*}(y, t)=-\left[\alpha \sigma_{1} \sum_{j=1}^{4} S_{3 j}^{*}(\tau) y_{j}+(1-\alpha) \sigma_{2} \sum_{j=1}^{4} S_{4 j}^{*}(\tau) y_{j}\right] \\
U_{2}^{*}(y, t)=-\sigma_{2} \sum_{j=1}^{4} S_{4 j}^{*}(\tau) y_{j},
\end{array}\right.
$$


where $Y(t)=y$ and $\tau=T-t$. In this case, $\left[S_{3 j}^{*}\right]_{j=1}^{4}$ and $\left[S_{4 j}^{*}\right]_{j=1}^{4}$ are the joint solution of the following differential equation system (which has been separated into three groups of equations)

$$
\begin{aligned}
& \left\{\begin{aligned}
\frac{\mathrm{d}}{\mathrm{d} \tau} S_{33}(\tau)= & 1-\alpha^{2} \sigma_{1}^{2} S_{33}^{2}(\tau)-2 \alpha(1-\alpha) \sigma_{1} \sigma_{2} S_{34}(\tau) S_{33}(\tau) \\
& -[\alpha(\alpha-2)+2] \sigma_{2}^{2} S_{34}^{2}(\tau) \\
\frac{\mathrm{d}}{\mathrm{d} \tau} S_{44}(\tau)= & 1-\alpha^{2} \sigma_{1}^{2} S_{34}^{2}(\tau)-2 \alpha(1-\alpha) \sigma_{1} \sigma_{2} S_{44}(\tau) S_{34}(\tau) \\
& -[\alpha(\alpha-2)+2] \sigma_{2}^{2} S_{44}^{2}(\tau) \\
\frac{\mathrm{d}}{\mathrm{d} \tau} S_{34}(\tau)= & -\alpha^{2} \sigma_{1}^{2} S_{34}(\tau) S_{33}(\tau)-\alpha(1-\alpha) \sigma_{1} \sigma_{2}\left[S_{44}(\tau) S_{33}(\tau)+S_{34}^{2}(\tau)\right] \\
& -[\alpha(\alpha-2)+2] \sigma_{2}^{2} S_{44}(\tau) S_{34}(\tau),
\end{aligned}\right. \\
& \int \frac{\mathrm{d}}{\mathrm{d} \tau} S_{13}(\tau)=-a S_{13}(\tau)-S_{33}(\tau)-\alpha^{2} \sigma_{1}^{2} S_{33}(\tau) S_{13}(\tau) \\
& -\alpha(1-\alpha) \sigma_{1} \sigma_{2}\left[S_{34}(\tau) S_{13}(\tau)+S_{33}(\tau) S_{14}(\tau)\right] \\
& -[\alpha(\alpha-2)+2] \sigma_{2}^{2} S_{14}(\tau) S_{34}(\tau), \\
& \frac{d}{\mathrm{~d} \tau} S_{14}(\tau)=-a S_{14}(\tau)-S_{34}(\tau)-\alpha^{2} \sigma_{1}^{2} S_{34}(\tau) S_{13}(\tau) \\
& -\alpha(1-\alpha) \sigma_{1} \sigma_{2}\left[S_{44}(\tau) S_{13}(\tau)+S_{34}(\tau) S_{14}(\tau)\right] \\
& -[\alpha(\alpha-2)+2] \sigma_{2}^{2} S_{44}(\tau) S_{14}(\tau), \\
& \int \frac{\mathrm{d}}{\mathrm{d} \tau} S_{23}(\tau)=-b S_{23}(\tau)-S_{34}(\tau)-\alpha^{2} \sigma_{1}^{2} S_{33}(\tau) S_{23}(\tau) \\
& -\alpha(1-\alpha) \sigma_{1} \sigma_{2}\left[S_{34}(\tau) S_{23}(\tau)+S_{33}(\tau) S_{24}(\tau)\right] \\
& -[\alpha(\alpha-2)+2] \sigma_{2}^{2} S_{34}(\tau) S_{24}(\tau) \text {, } \\
& \frac{\mathrm{d}}{\mathrm{d} \tau} S_{24}(\tau)=-b S_{24}(\tau)-S_{44}(\tau)-\alpha^{2} \sigma_{1}^{2} S_{34}(\tau) S_{23}(\tau) \\
& -\alpha(1-\alpha) \sigma_{1} \sigma_{2}\left[S_{44}(\tau) S_{23}(\tau)+S_{34}(\tau) S_{24}(\tau)\right] \\
& -[\alpha(\alpha-2)+2] \sigma_{2}^{2} S_{44}(\tau) S_{24}(\tau) \text {, }
\end{aligned}
$$

with the conditions $S_{33}(0)=S_{44}(0)=1$, and $S_{14}(0)=S_{24}(0)=S_{34}(0)=$ $S_{13}(0)=S_{23}(0)=0$. We shall provide solutions $\left[S_{3 j}^{*}\right]_{j=1}^{4},\left[S_{4 j}^{*}\right]_{j=1}^{4}$, in the particular case, for which

$$
\alpha^{2} \sigma_{1}^{2}=q, \text { and, } \sigma_{2}^{2}=q\left[(1-\alpha)^{2}+1\right]^{-1}, \quad \sigma_{1}, \sigma_{2}>0, \quad 0<\alpha<1,
$$

hold for some $q>0$. Then, for the first three-equation group, $S_{33} \equiv S_{44}$ on $[0, T]$, having instead of three equations, just the following two:

$$
\left\{\begin{array}{l}
\frac{\mathrm{d}}{\mathrm{d} \tau} S_{33}(\tau)=1-q\left[S_{33}^{2}(\tau)+S_{34}^{2}(\tau)\right]-\frac{2 q(1-\alpha)}{\sqrt{(1-\alpha)^{2}+1}} S_{33}(\tau) S_{34}(\tau), \\
\frac{\mathrm{d}}{\mathrm{d} \tau} S_{34}(\tau)=-\frac{q(1-\alpha)}{\sqrt{(1-\alpha)^{2}+1}}\left[S_{33}^{2}(\tau)+S_{34}^{2}(\tau)\right]-2 q S_{33}(\tau) S_{34}(\tau),
\end{array}\right.
$$


If we denote $C_{0}^{2}=q-\left[q(1-\alpha) / \sqrt{(1-\alpha)^{2}+1}\right]$, then from (4.11), we have

$$
\left\{\begin{array}{l}
\frac{\mathrm{d}}{\mathrm{d} \tau} S_{33}(\tau)=1-q\left[S_{33}(\tau)+S_{34}(\tau)\right]^{2}+2 C_{0}^{2} S_{33}(\tau) S_{34}(\tau) \\
\frac{\mathrm{d}}{\mathrm{d} \tau} S_{34}(\tau)=-\frac{q(1-\alpha)}{\sqrt{(1-\alpha)^{2}+1}}\left[S_{33}(\tau)+S_{34}(\tau)\right]^{2}-2 C_{0}^{2} S_{33}(\tau) S_{34}(\tau)
\end{array}\right.
$$

if now $C_{1}^{2}=q+\left[q(1-\alpha) / \sqrt{(1-\alpha)^{2}+1}\right]$, then from (4.11), we may also write

$$
\left\{\begin{array}{l}
\frac{\mathrm{d}}{\mathrm{d} \tau} S_{33}(\tau)=1-q\left[S_{33}(\tau)-S_{34}(\tau)\right]^{2}-2 C_{1}^{2} S_{33}(\tau) S_{34}(\tau) \\
\frac{\mathrm{d}}{\mathrm{d} \tau} S_{34}(\tau)=-\frac{q(1-\alpha)}{\sqrt{(1-\alpha)^{2}+1}}\left[S_{33}(\tau)-S_{34}(\tau)\right]^{2}-2 C_{1}^{2} S_{33}(\tau) S_{34}(\tau) .
\end{array}\right.
$$

Setting $F_{1}(\tau)=S_{33}(\tau)+S_{34}(\tau)$ and $F_{0}(\tau)=S_{33}(\tau)-S_{34}(\tau)$, we obtain from (4.12) and (4.13) that

$$
\frac{\mathrm{d}}{\mathrm{d} t} F_{k}(\tau)=C_{k}^{2}\left[\frac{1}{C_{k}^{2}}-F_{k}^{2}(\tau)\right], \quad F_{k}(0)=1, \quad k=0,1 .
$$

The solutions of (4.14) are found as

$$
F_{k}(\tau)=\frac{1}{C_{k}}\left[\frac{\left(1+C_{k}\right)\left(1-C_{k}\right)^{-1} e^{2 C_{k} t}-1}{\left(1+C_{k}\right)\left(1-C_{k}\right)^{-1} e^{2 C_{k} t}+1}\right], \quad k=0,1,
$$

from which

$$
S_{44}^{*}(\tau)=S_{33}^{*}(\tau)=\frac{1}{2}\left[F_{0}(\tau)+F_{1}(\tau)\right], \quad S_{34}^{*}(\tau)=\frac{1}{2}\left[F_{1}(\tau)-F_{0}(\tau)\right] .
$$

Now, define a matrix $H^{*}(\tau ; c)$, for $c=a, b$, with entries

$$
\left\{\begin{array}{l}
H_{11}^{*}(\tau ; c)=H_{22}^{*}(\tau ; c)=-c-q S_{33}^{*}(\tau)-\frac{q(1-\alpha)}{\sqrt{(1-\alpha)^{2}+1}} S_{34}^{*}(\tau), \\
H_{12}^{*}(\tau ; c)=H_{21}^{*}(\tau ; c)=-\frac{q(1-\alpha)}{\sqrt{(1-\alpha)^{2}+1}} S_{33}^{*}(\tau)-q S_{34}^{*}(\tau),
\end{array}\right.
$$

so that

$$
\frac{\mathrm{d}}{\mathrm{d} t}\left(\begin{array}{c}
S_{13}(\tau) \\
S_{14}(\tau)
\end{array}\right)=H^{*}(\tau ; a)\left(\begin{array}{c}
S_{13}(\tau) \\
S_{14}(\tau)
\end{array}\right)-\left(\begin{array}{c}
S_{33}^{*}(\tau) \\
S_{34}^{*}(\tau)
\end{array}\right)
$$

and

$$
\frac{\mathrm{d}}{\mathrm{d} t}\left(\begin{array}{l}
S_{23}(\tau) \\
S_{24}(\tau)
\end{array}\right)=H^{*}(\tau ; b)\left(\begin{array}{l}
S_{23}(\tau) \\
S_{24}(\tau)
\end{array}\right)-\left(\begin{array}{l}
S_{34}^{*}(\tau) \\
S_{44}^{*}(\tau)
\end{array}\right)
$$

Also, define the functions

$$
Q^{*}(\tau ; c)=-\int_{0}^{\tau} H_{11}^{*}(u ; c) \mathrm{d} u, \quad R^{*}(\tau ; c)=-\int_{0}^{\tau} H_{12}^{*}(u ; c) \mathrm{d} u, \quad c=a, b .
$$


Then, the solutions of (4.15) and (4.16) are found as

$$
\left\{\begin{aligned}
S_{13}^{*}(\tau)= & \int_{0}^{\tau} S_{34}^{*}(s) e^{-\left[Q^{*}(\tau ; a)-Q^{*}(s ; a)\right]} \sinh \left[R^{*}(\tau ; a)-R^{*}(s ; a)\right] \mathrm{d} s \\
& -\int_{0}^{\tau} S_{33}^{*}(s) e^{-\left[Q^{*}(\tau ; a)-Q^{*}(s ; a)\right]} \cosh \left[R^{*}(\tau ; a)-R^{*}(s ; a)\right] \mathrm{d} s, \\
S_{14}^{*}(\tau)= & \int_{0}^{\tau} S_{33}^{*}(s) e^{-\left[Q^{*}(\tau ; a)-Q^{*}(s ; a)\right]} \sinh \left[R^{*}(\tau ; a)-R^{*}(s ; a)\right] \mathrm{d} s \\
& -\int_{0}^{\tau} S_{34}^{*}(s) e^{-\left[Q^{*}(\tau ; a)-Q^{*}(s ; a)\right]} \cosh \left[R^{*}(\tau ; a)-R^{*}(s ; a)\right] \mathrm{d} s, \\
S_{23}^{*}(\tau)= & \int_{0}^{\tau} S_{44}^{*}(s) e^{-\left[Q^{*}(\tau ; b)-Q^{*}(s ; b)\right]} \sinh \left[R^{*}(\tau ; b)-R^{*}(s ; b)\right] \mathrm{d} s \\
& -\int_{0}^{\tau} S_{34}^{*}(s) e^{-\left[Q^{*}(\tau ; b)-Q^{*}(s ; b)\right]} \cosh \left[R^{*}(\tau ; b)-R^{*}(s ; b)\right] \mathrm{d} s, \\
S_{24}^{*}(\tau)= & \int_{0}^{\tau} S_{34}^{*}(s) e^{-\left[Q^{*}(\tau ; b)-Q^{*}(s ; b)\right]} \sinh \left[R^{*}(\tau ; b)-R^{*}(s ; b)\right] \mathrm{d} s \\
& -\int_{0}^{\tau} S_{44}^{*}(s) e^{-\left[Q^{*}(\tau ; b)-Q^{*}(s ; b)\right]} \cosh \left[R^{*}(\tau ; b)-R^{*}(s ; b)\right] \mathrm{d} s .
\end{aligned}\right.
$$

For small $\tau$ we may provide approximations, using the following set of relations:

$$
\left\{\begin{array}{l}
\left(\text { i) } \quad Q^{*}(\tau ; c)-Q^{*}(s ; c) \approx-H_{11}^{*}(\tau ; c)(\tau-s), \quad c=a, b,\right. \\
(\text { ii }) \sinh \left[R^{*}(\tau ; c)-R^{*}(s ; c)\right] \approx R^{*}(\tau ; c)-R^{*}(s ; c), \quad c=a, b, \\
\left(\text { iii) } \cosh \left[R^{*}(\tau ; c)-R^{*}(s ; c)\right] \approx 1+\frac{1}{2}\left[R^{*}(\tau ; c)-R^{*}(s ; c)\right]^{2}, \quad c=a, b,\right. \\
(\text { iv }) R^{*}(\tau ; c)-R^{*}(s ; c) \approx-H_{12}^{*}(\tau ; c)(\tau-s), \quad c=a, b .
\end{array}\right.
$$

Applying $(i)-(i v)$, we may obtain an approximation, for instance, to $S_{13}^{*}$, as follows:

$$
\begin{aligned}
S_{13}^{*}(\tau) \approx & -\int_{0}^{\tau} S_{34}^{*}(s) e^{H_{11}^{*}(\tau ; a)(\tau-s)} H_{12}^{*}(\tau ; a)(\tau-s) \mathrm{d} s \\
& -\int_{0}^{\tau} S_{33}^{*}(s) e^{H_{11}^{*}(\tau ; a)(\tau-s)}\left\{1+\frac{1}{2}\left[H_{12}^{*}(\tau ; a)(\tau-s)\right]^{2}\right\} \mathrm{d} s .
\end{aligned}
$$

\section{Summary and Conclusions}

For a number of stochastic demand models, when production and inventory costs are quadratic, we have provided via the LQG approach, closed-form and approximate solutions for optimal production-inventory policies in a dynamic monopoly model. We found the following delimitations of our work: more work has to be done for multidimensional models when the demand vector consists of dependent components. The assumption about admissible controls 
to be Lipschitz continuous in the initial state rules out "bang-bang" controls. No lower limit for the inventory level and the production rate are assumed. Finally, $A, B, C, P, Q$ and $M$ are all time-invariant matrices.

\section{References}

Arnold, L. (1982). Stochastic Differential Equations. Wiley, New York.

Athans, M. (1971). Special Issue on the Linear-Quadratic-Gaussian Estimation and the Control Problem. IEEE Transactions on Automatic Control, AC-11, pp. 527-596.

Bather, J. A. (1968). A Diffusion Model for the Control of a Dam. Journal of Applied Probability, 5, pp. 51-71.

Bensoussan, A., S. P. Sethi, R. Vickson, and N. Derzko (1984). Stochastic Production Planning with Production Constraints. SIAM, Journal Control and Optimization, 22(6), pp. $920-935$.

Bensoussan, A. (1982). Stochastic Control by Functional Analysis Methods. North-Holland. Elliott, R. J. (1982). Stochastic Calculus and Applications. Springer-Verlag, New York.

Fleming, W. H., and R. W. Rishel (1975). Deterministic and Stochastic Optimal. Springer Verlag, New York.

Hernández, D. B. (1987). La Ecuación de Hamilton-Jacobi-Bellman y Algunas Aplicaciones. Miscelánea Matemática, 16, pp. 50-78.

Hirsh, M. W. (1974). Differential Equations, Dynamical Systems, and Linear Algebra. Academic Press, New York.

Pliska, S. R. (1975). A Diffusion Process Model for the Optimal Operation of a Reservoir System. Journal of Applied Probability, 12, pp. 859-863. 